\title{
MR Sönümleyicili Yarı Aktif Ayarlı Kütle Sönümleyicisinin Uyarlamalı Kontrolü
}

\author{
Mahmut Paksoy ${ }^{1 *}$, Hüseyin Aggümüş ${ }^{2}$ \\ ${ }^{1 *}$ Selçuk Üniversitesi, Akşehir Mühendislik ve Mimarlık Fakültesi, Makine Mühendsiliği Bölümü, Konya, Türkiye, (ORCID: 0000-0002-3171-6410), \\ mahmut.paksoy@selcuk.edu.tr \\ ${ }^{2}$ Şırnak Üniversitesi, Şırnak Meslek Yüksekokulu, Makine ve Metal Teknolojileri Bölümü, Şırnak, Türkiye (ORCID: 0000-0002-7158-677X), \\ haggumus@sirnak.edu.tr
}

(İlk Geliş Tarihi 8 Kasım 2021 ve Kabul Tarihi 3 Ocak 2021)

(DOI: 10.31590/ejosat.1020498)

ATIF/REFERENCE: Paksoy, M. \& Aggümüş, H. (2022). MR Sönümleyicili Yarı Aktif Ayarlı Kütle Sönümleyicisinin Uyarlamalı Kontrolü. Avrupa Bilim ve Teknoloji Dergisi, (33), 68-73.

$\ddot{\mathbf{O z}}$

Bu çalışmada çok serbestlik dereceli bir bina modelinin bozucu girişler etkisindeki cevaplarını iyileştirmek için yarı-aktif ayarlı kütle sönümleyicisi (YAKS) kullanılmıştır. Tasarlanan YAKS'de yarı-aktifliği sağlayan kontrol elemanı olarak Magnetorheological (MR) sönümleyici kullanılmıştır. MR sönümleyiciler uygulanan gerilimle sönüm oranı ayarlanabilen kontrol elemanlarıdır. Uygun bir kontrol algoritmasıyla bu gerilim değerlerini belirlemek mümkündür. MR sönümleyici oldukça nonlineer bir karaktere sahiptir ve çalışması esnasında ısınır. Bu ısınmadan dolayı sahip olduğu parametre değerleri çalışma sırasında değişebilir. Bu yüzden hem nonlineerliklerin hem de parametrik belirsizliklerin üstesinden gelebilecek bir kontrolör kullanılması performansı arttıracaktır. Bu amaçla, bu çalışmada nonlineer uyarlamalı kontrol algoritması tasarlanmıştır. Kontrolcü performansı, pasif kontrol uygulaması olan ayarlı kütle sönümleyicisi (AKS) ile YAKS karşılaştırılarak incelenmiştir. Sonuçlar, uyarlamalı kontrol uygulamasıyla YAKS'nin AKS'den daha iyi performans gösterdiğini kanıtlamıştır.

Anahtar Kelimeler: Uyarlamalı kontrol, Yapısal titreşim kontrolü, MR sönümleyici, Yarı aktif kütle sönümleyici (YAKS).

\section{Adaptive Control of Semi Active Tuned Mass Damper with MR Damper}

\begin{abstract}
In this study, a semi active tuned mass damper (STMD) is used to improve the responses of a multi-degree-of-freedom building model under disturbance input. In the designed STMD, MR damper is employed in the system as a semi active control element. MR damper is a control element whose damping force can be adjusted to applied voltage. It is possible to determine the required voltage with a convenient control algorithm. Required voltage can be determined with a convenient control algorithm. The MR damper has a nonlinear hysteresis character, and its temperature increases during operation. Due to this temperature variance, its parameters may change during operation. Therefore, in order to obtain better performance, the designed controller must be able to overcome both nonlinearity and parametric uncertainties. That's why a nonlinear adaptive control algorithm is designed in this study. For analysis of controller performance, TMD and STMD results are compared. The results showed that the performance of STMD, which is the application of adaptive control better than the TMD situation.
\end{abstract}

Keywords: Adaptive control, Structural vibration control, MR damper, Semi active tuned mass damper (STMD).

\footnotetext{
*Sorumlu Yazar: mahmut.paksoy@selcuk.tr
} 


\section{Giriş}

Yapısal sistemlerin bozucu girişlerin (deprem, rüzgâr vs.) etkisinden korunması düşüncesi, bu sistemlerde birçok kontrol uygulamasının geliştirilmesine neden olmuştur. Ayarlı kütle sönümleyicileri (AKS) bunların en sık kullanılanlarındandır. Temel olarak aktif, pasif ve yarı-aktif olmak üzere üç farklı kontrol uygulaması yaygın olarak kullanılmaktadır. Ayarlı kütle sönümleyicileri pasif sistem olarak tasarlanmasına karşın, pasif duruma göre daha iyi sonuçlar veren aktif ve yarı-aktif sistemler olarak da tasarlanabilirler.

AKS'lerin aktif kontrolü ile ilgili yapılan birçok çalışma vardır [1-4]. AKS'ler ile ilgili yapılan aktif kontrol çalışmalarında yüksek performans elde edilmesine rağmen gerek maliyetlerinin fazla oluşu, gerekse güvenilirlik ile ilgili duyulan endişeler olumsuz yönler olarak karşımıza çıkmaktadır. AKS'lerin yarı-aktif uygulamaları ise, aktif kontrolden maliyet ve güvenlik olarak daha avantajlı olması ve pasif kontrolün performansının arttırılması bakımından daha avantajlıdır [5].

MR sönümleyiciler yapısal sistemlerde yaygın olarak kullanılan yarı-aktif kontrol elemanlarıdır [5-16]. AKS'lerde sönüm elemanı yerine MR sönümleyicilerin kullanılmasıyla YAKS'ler elde edilir. Literatürde, MR sönümleyicili YAKS ile ilgili simülasyon çalışmaları [6-11], deneysel çalışmalar [12,13] ve hibrit simülasyon çalışması yapılmıştır $[15,16]$. Tek serbestlik dereceli $[6,11,13]$ ve çok serbestlik dereceli $[5,7-10,12,15,16]$ olarak yapılan bu çalışmalar, Groundhook kontrol [5], Nedensel alt optimal kontrol [6], Kırpılmış optimal kontrol [7], LQR kontrol [7, 8], Bulanık mantıklı kontrol [9], Bang-bang kontrol [10], Model tabanlı ileri beslemeli kontrol [13], Dayanıklı Hळ kontrol [15], Skyhook kontrol [16], gibi basit ya da karmaşık kontrol yöntemleriyle gerçekleştirilmiştir.

Laboratuvarda kullanılan deney sistemlerinde kullanılan yapıların parametreleri gerçeğe çok yakın olarak belirlenebilse de gerçek yapıların karakteristiklerini belirleyen parametrelerin doğru bir şekilde belirlenmesi zor olabilir. Ayrıca, sistem parametreleri kullanıma bağlı olarak zaman içerinde değişiklik gösterebilir. Eyleyici olarak kullanılan MR sönümleyici gibi nonlineer histerezis karaktere sahip yarı aktif elemanların modellenmesindeki parametre belirsizlikleri kontrolör performansını da direk olarak etkilemektedir [17]. Buna ek olarak çalışma sırasında ısınmadan dolayı MR sönümleyicinin sahip olduğu parametreler değişiklik gösterir [18].

Sonuç olarak, yukarıda bahsedildiği gibi, yapısal sistemler ve MR sönümleyiciler parametrik belirsizliklere sahip sistemlerdir. Ayrıca, MR sönümleyiciler nonlineer histerezis karaktere sahiptir. Literatür incelendiğinde ise YAKS tasarımında uyarlamalı kontrol yönteminin ise daha önce kullanılmadığı görülmektedir. Tüm bu durumlar göz önüne alınarak, parametrik belirsizlikerin üstesinden gelebilecek ve MR sönümleyicinin nonlineer dinamiği ile uyumlu olarak bu çalışmada nonlineer uyarlamalı kontrol yöntemi kullanılmıştır. Sonuçlar çok serbestlik dereceli (ÇSD) bir bina modeli kullanılarak, MATLAB-Simulink programı yardımıyla gerçekleştirilen simülasyonlar ile incelenmiştir.

\section{Materyal ve Metot}

\subsection{Bina Modeli}

Deprem ve rüzgâr gibi harici bozucular etkisindeki yapılarda meydana gelen yanal hareket, yapıda yüksek gerilmelere neden olur [19]. Bu nedenle yapilarda yanal hareketin incelenmesi kritik öneme sahiptir. Gerek yapıya ait bir kısım (örneğin kirişler) [24] gerekse yapının tamamının kontrolü ile ilgili çalışmalar araştırmacıların ilgisini çekmektedir. $\mathrm{Bu}$ çalışmadaki yarı aktif kontrol uygulaması, yapının yanal hareketi dikkate alınarak Şekil 1'de görülen on katlı bir bina modelinin en üst katına YAKS eklenerek gerçekleştirilmiştir. Modele ait genel denklem ifadesi Eşitlik 1'de verilmiştir.
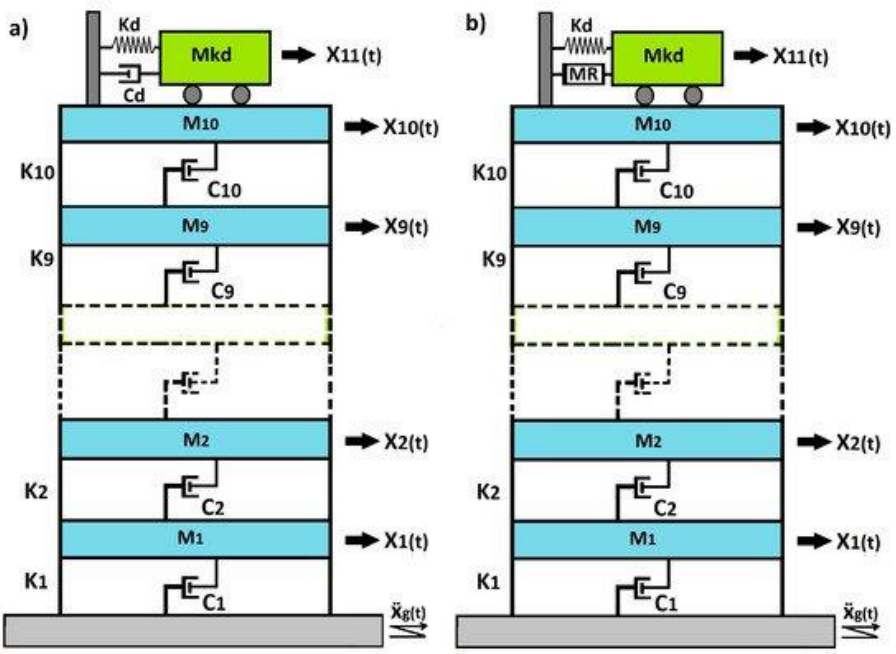

Şekil 1 (a) AKS'li bina modeli (b) YAKS'li bina modeli [15]

Figure 1 (a) Structural model with TMD (b) Structural model with STMD [15]

$$
M_{s} \ddot{x}(t)+C_{s} \dot{x}(t)+K_{s} x(t)=-H_{s} f(t)-M_{s} L \ddot{x}_{g}
$$

Burada sistem matrisleri olan $M_{s}, C_{s}$ ve $K_{s}$ sirasiyla $\mathfrak{R}^{11 x 11}$ boyutlu kütle, sönüm ve rijitlik olarak ifade edilir. $f(t) \mathrm{MR}$ sönümleyici sönüm kuvveti, $L$ tahrik giriş vektörü, $H_{s}$ kontrolün uygulandığı yeri gösteren matris ve $\ddot{x}_{g}$ tahrik olarak ifade edilir. $\ddot{x}(t), \dot{x}(t)$ ve $x(t)$ vektörleri ise sirasıyla $\mathfrak{R}^{11 x 1}$ boyutlu ivme, hız ve yer değiştirme vektörleridir. Yer değiştirme vektörü $x=\left[\begin{array}{lllll}x_{1} & x_{2} & \cdots & x_{10} & x_{d}\end{array}\right]$ bozucu tahrik giriş vektörü $L=\left[\begin{array}{lllll}1 & 1 & \cdots & 1 & 1\end{array}\right]^{T}$ ' dir. Kontrolcünün yerini gösteren vektör $H_{s}=\left[\begin{array}{lllll}0 & 0 & \cdots & 1 & -1\end{array}\right]^{T}$ şeklindedir. AKS'nin parametrelerini hesaplamak için, optimum frekans oranı Eşitlik 2'de, optimum sönüm oranı ise Eşitlik 3 'te verilmiştir [20].

$$
\begin{gathered}
f_{\text {opt }}=\frac{1}{1+\mu}\left[1-\xi \sqrt{\frac{\mu}{1+\mu}}\right] \\
\xi_{\text {opt }}=\frac{\xi}{1+\mu}+\sqrt{\frac{\mu}{1+\mu}}
\end{gathered}
$$

Burada $\mu$ kütle oranıdır ve AKS'nin kütlesinin binanın toplam kütlesine oranıdır. $\mathrm{Bu}$ çalışmada $\mu$ değeri 0.02 olarak alınmıştır. Bina modeline ait parametreler $\mathrm{m}_{1-10}=72 \times 10^{3} \mathrm{~kg}$, $\mathrm{k}_{1-10}=13 \times 10^{7} \mathrm{~N} / \mathrm{m}, \quad \mathrm{c}_{1-10}=1.24 \times 10^{6} \mathrm{Ns} / \mathrm{m}^{\prime} \operatorname{dir}$ [15]. AKS'nin sırasıyla kütle, sönüm ve rijitlik değerleri $m_{d}=21600 \mathrm{~kg}, c_{d}=$ $53016 \mathrm{Ns} / \mathrm{m}, \mathrm{k}_{\mathrm{d}}=812718 \mathrm{~N} / \mathrm{m}$ olarak elde edilmiştir. Bina modeline uygulanan tahrik girişi, $0.035 \mathrm{~m} / \mathrm{s}^{2}$ genliğinde ve 
binanın tüm doğal frekanslarının toplamından oluşmaktadır. Sisteme 30 saniye süresince uygulanan tahrik girişi Şekil 2'de verilmiştir.

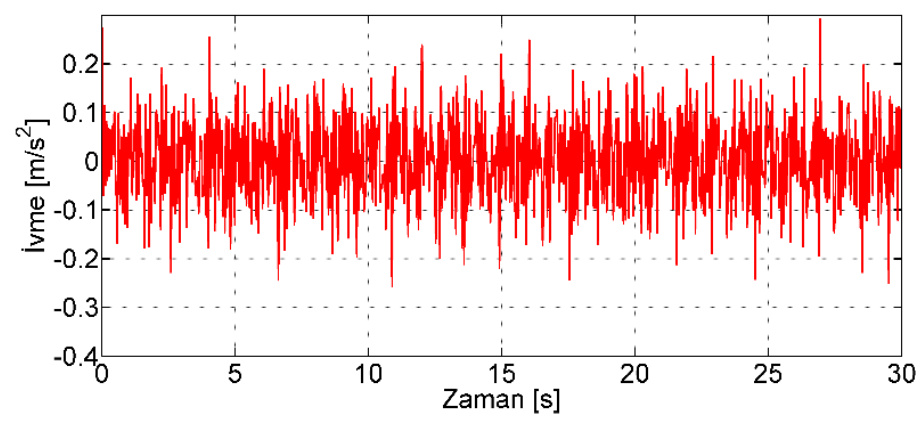

Şekil 2 Sisteme etki eden tahrik girişi

Figure 2 Applied excitation input to the system

\subsection{Yarı Aktif Uyarlamalı Kontrolör Tasarımı}

$\mathrm{Bu}$ çalışmada tasarlanan uyarlamalı kontrol algoritması ve simülasyon çalışmaları için $\mathrm{MR}$ sönümleyicinin dinamik davranışını iyi bir şekilde yansıtabilen matematiksel modeline ihtiyaç vardır. Literatürde MR sönümleyicinin nonlineer karakterini uygun şekilde yansıtabilen birçok MR sönümleyici modeli vardır (Modified Bouc Wen, Dahl etc.) [21]. Uyarlamalı kontrol tasarımında ve kararlılık analizi kısmında oldukça karmaşık denklemler ortaya çıkmaktadır. $\mathrm{Bu}$ yüzden, uygulanabilirliği arttırmak için, basit matematiksel yapıya sahip ve gerçek MR sönümleyici cevabına yakın sonuç verebilen bir MR sönümleyici matematiksel modeli tercih etmek uygun olacaktır. Bu faktörler göz önünde bulundurularak bu çalışmada, nispeten daha sade denklemlere sahip olan Lugre MR sönümleyici matematiksel modeli tercih edilmiştir [22]. Lugre modeli denklemleri aşağıdaki gibidir;

$$
\begin{gathered}
f=\sigma_{a} z+\sigma_{0} z v+\sigma_{1} \dot{z}+\sigma_{2} \dot{x}_{r}+\sigma_{b} \dot{x}_{r} v \\
\dot{z}=\dot{x}_{r}-a_{0}\left|\dot{x}_{r}\right| z
\end{gathered}
$$

Yapısal sistem için tasarlanan uyarlamalı kontrolcünün amacı, binayı depremin yıkıcı etkisinden korurken kapalı sistemin kararlılığını da sağlamaktır. Bu amaçla Lugre modeli kullanılarak uyarlamalı kontrolcü tasarlanmış ve kapalı çevrim sistemin kararlılığı Lyapunov temelli kararlılık analizi ile gerçekleştirilmiştir. Burada binayı depremin yıkıcı etkisinden korumanın yolu, bina katlarının yer değiştirmelerinin sıfır olmasını sağlamaktır. Bu amaçla sıfıra gitmesini istediğimiz sisteme ait yer değiştirme ve hızları içeren hata dinamiği ve zamana göre türevi aşağıdaki gibi tanımlanmıştır;

$$
r=\dot{x}+\lambda x, \dot{r}=\ddot{x}+\lambda \dot{x}
$$

Eşitlik 4 ve Eşitlik 5 'in yapısı incelendiğinde Eşitlik 5 'in Eşitlik 4'ün içerisine yazılabileceği görülmektedir. $\mathrm{Bu}$ işlem yapıldığında MR sönümleyicinin sönüm kuvvetini veren denklem aşağıdaki formu alacaktır;

$$
f=\sigma_{a} z+\sigma_{0} z v-\sigma_{1} a_{0}\left|\dot{x}_{r}\right| z+\left(\sigma_{1}+\sigma_{2}\right) \dot{x}_{r}+\sigma_{b} \dot{x}_{r} v
$$

Burada $x_{r}$ MR sönümleyici rölatif yer değiştirmesi, $v$ MR sönümleyiciye gönderilen gerilim, $z$ MR sönümleyici iç değişkeni ve $f$ ise MR sönümleyici değişken sönüm kuvvetidir. Diğer parametreler ise MR sönümleyicinin histeresiz karakterini tanımlamada kullanılan sabitlerdir. Bu çalışmada Lugre model e-ISSN: 2148-2683 parametreleri ve yapısal sistemin parametrelerinin değerinin tam olarak bilinmediği varsayılmıştır. $\mathrm{Bu}$ yüzden kontrolör tasarımında parametrelerin tahmin değerleri kullanılmalıdır. Bir parametrenin tahmin ve tahmin hatasi ifadesi sirasiyla parametrenin üzerine “^” ve “ ” işareti konularak gösterilmiştir. Dolayısıyla MR sönümleyici sönüm kuvvetinin tahmini aşağıdaki gibidir;

$$
\hat{f}=\hat{\sigma}_{a} \hat{z}-\hat{\sigma}_{1} a_{0}\left|\dot{x}_{r}\right| \hat{z}+\left(\hat{\sigma}_{1}+\hat{\sigma}_{2}\right) \dot{x}_{r}+\left(\hat{\sigma}_{0} \hat{z}+\hat{\sigma}_{b} \dot{x}_{r}\right) v
$$

Eşitlik 6'da verilen hata dinamiğinin zaman göre türevi soldan kütle matrisiyle çarpılırsa Eşitlik 9 aşağıdaki gibi elde edilir;

$$
\begin{aligned}
M \dot{r} & =M \lambda \dot{x}-K x-M L \ddot{x}_{g}-H f \\
& =Y \phi-H f
\end{aligned}
$$

Burada $Y \in \mathfrak{R}^{11 \times 22}$ boyutlu bilinen ya da ölçülebilen terimlerden oluşan matris, $\quad \phi=\in \mathfrak{R}^{22 x 1}$ ise bilinmeyen terimlerden oluşan matristir. $Y$ ve $\phi$ aşağıdaki gibidir;

$$
\begin{gathered}
Y=\left[\begin{array}{ll}
Y_{M} & Y_{K}
\end{array}\right] \\
Y_{M}=\left[\begin{array}{cccc}
\lambda_{1} \dot{x}_{1}-\ddot{x}_{g} & 0 & \cdots & 0 \\
0 & \lambda_{2} \dot{x}_{2}-\ddot{x}_{g} & \cdots & 0 \\
\vdots & \vdots & \ddots & \vdots \\
0 & 0 & \cdots & \lambda_{11} \dot{x}_{d}-\ddot{x}_{g}
\end{array}\right] \\
Y_{K}=\left[\begin{array}{cccc}
x_{1} & x_{1}-x_{2} & \cdots & 0 \\
0 & x_{2}-x_{1} & \cdots & 0 \\
\vdots & \vdots & \ddots & \vdots \\
0 & 0 & \cdots & x_{d}-x_{10}
\end{array}\right] \\
\phi=\left[\begin{array}{llll}
\phi_{M} & \phi_{K}
\end{array}\right]^{T} \\
\phi_{M}=\left[\begin{array}{llll}
m_{1} & m_{2} & \cdots & m_{d}
\end{array}\right] \\
\phi_{K}=\left[\begin{array}{llll}
k_{1} & k_{2} & \cdots & k_{d}
\end{array}\right]
\end{gathered}
$$

$H \hat{f}$ terimi Eşitlik 9'a eklenip çıkarılır ve $\chi, u_{x}, \rho$ and $\tilde{\theta}$ terimleri aşağıdaki gibi tanımlanırsa Eşitlik 17 aşağıdaki formu alacaktır.

$$
\begin{gathered}
\chi=-\hat{\sigma}_{a} \hat{z}+\hat{\sigma}_{1} a_{0}\left|\dot{x}_{r}\right| \hat{z}-\left(\hat{\sigma}_{1}+\hat{\sigma}_{2}\right) \dot{x}_{r} \\
u_{x}=\left(\hat{\sigma}_{0} \hat{z}+\hat{\sigma}_{b} \dot{x}_{r}\right) v \\
\rho=\left[\begin{array}{ll}
\dot{x}_{r} & \dot{x}_{r} v
\end{array}\right], \quad \tilde{\theta}=\left[\begin{array}{ll}
\left(\tilde{\sigma}_{1}+\tilde{\sigma}_{2}\right) & \tilde{\sigma}_{b}
\end{array}\right]^{T} \\
M \dot{r}=Y \phi+H \chi-H u_{x}-H \rho \tilde{\theta} \\
-H\left[\sigma_{a} z-\hat{\sigma}_{a} \hat{z}-\sigma_{1} a_{0}\left|\dot{x}_{r}\right| z+\hat{\sigma}_{1} a_{0}\left|\dot{x}_{r}\right| \hat{z}+\sigma_{0} z v-\hat{\sigma}_{0} \hat{z} v\right]
\end{gathered}
$$

Kontrolcünün yer değiştirmeleri sıfir yapması amacına uygun olarak Eşitlik 17 incelendiğinde $H u_{x}$ ifadesi Eşitlik 18'deki gibi seçilebilir. Dolayısıyla MR sönümleyiciye gönderilmesi gereken gerilim miktarı Eşitlik 19'daki formda olacaktır. Hesaplanan bu gerilim miktarı anlık olarak MR sönümleyiciye gönderilerek kapalı çevrim sistem elde edilmiş olur. Kapalı çevrim sistem genel yapısı Şekil 3 ’te verilmiştir. 


$$
\begin{gathered}
H u_{x}=K r+H \chi+Y \hat{\phi} \\
+H\left(-\hat{\sigma}_{a} \xi_{1}-\hat{\sigma}_{0} \xi_{2} v+\hat{\sigma}_{1} a_{0}\left|\dot{x}_{r}\right| \xi_{3}\right) \\
v=\frac{H^{-1}\left[K r+H \chi+Y \hat{\phi}+H\left(-\hat{\sigma}_{a} \xi_{1}+\hat{\sigma}_{1} a_{0}\left|\dot{x}_{r}\right| \xi_{3}\right)\right]}{\left(\hat{\sigma}_{0} \xi_{2}+\hat{\sigma}_{0} \hat{z}+\hat{\sigma}_{b} \dot{x}_{r}\right)}
\end{gathered}
$$

Burada $\xi_{1}, \xi_{2}$ ve $\xi_{3}$ yardımc filtrelerdir ve kararlılık analizi kısmında tanımlanacaklardır. Sonuç olarak kontrol sinyali sistem denklemleri içerisine eklenerek kapalı çevrim sistem denklemi Eşitlik 20'deki gibi elde edilir. Lugre modelindeki $z$ MR sönümleyici iç değişkenidir ve ölçülmesi mümkün değildir. Dolayısıyla gözlemleyici tasarlanmalıdır. $z$ için tasarlanan gözlemleyici denklemi Eşitlik 21'de verilmiştir.

$$
\begin{aligned}
& M \dot{r}=-K r+Y \tilde{\phi}-H \rho \tilde{\theta} \\
& +H\left[-\tilde{\sigma}_{a}\left(\hat{z}+\xi_{1}\right)-\sigma_{a}\left(\tilde{z}-\xi_{1}\right)\right] \\
& +H\left[-\tilde{\sigma}_{0} v\left(\hat{z}+\xi_{2}\right)-\sigma_{0} v\left(\tilde{z}-\xi_{2}\right)\right] \\
& +H\left[\tilde{\sigma}_{1} a_{0}\left|\dot{x}_{r}\right|\left(\hat{z}+\xi_{3}\right)+\sigma_{1} a_{0}\left|\dot{x}_{r}\right|\left(\tilde{z}-\xi_{3}\right)\right] \\
& \qquad \dot{\tilde{z}}=-a_{0}\left|\dot{x}_{r}\right| \tilde{z}
\end{aligned}
$$



Şekil 3 YAKS genel sistem yapısı

Şekil 3 STMD Overall system structure

\subsubsection{Yarı Aktif Uyarlamalı Kontrolör Tasarımı}

Kapalı çevrim sistemin kararlılığını incelemek amacıyla aşağıdaki negatif olmayan Lyapunov fonksiyonu seçilmiş̧tir.

$$
\begin{aligned}
& V=\frac{1}{2} r^{T} M r+\frac{1}{2} \tilde{z}^{2}+\frac{1}{2} \tilde{\phi}^{T} \Gamma_{\phi}^{-1} \tilde{\phi}+\frac{1}{2} \tilde{\theta}^{T} \Gamma^{-1} \tilde{\theta} \\
& +\frac{1}{2} \frac{1}{\gamma_{1}} \tilde{\sigma}_{a}^{2}+\frac{1}{2} \frac{1}{\gamma_{2}} \tilde{\sigma}_{0}^{2}+\frac{1}{2} \frac{1}{\gamma_{3}}\left(\tilde{\sigma}_{1} a_{0}\right)^{2}+\frac{1}{2} \sigma_{a}\left(\tilde{z}-\xi_{1}\right)^{2} \\
& +\frac{1}{2} \sigma_{0}\left(\tilde{z}-\xi_{2}\right)^{2}+\frac{1}{2} \sigma_{1} a_{0}\left(\tilde{z}-\xi_{3}\right)^{2}
\end{aligned}
$$

Burada $\Gamma_{\phi}$, ve pozitif tanıml katsayı matrisi. $\gamma_{1}, \gamma_{2}$ ve $\gamma_{3}$ ise pozitif katsayılardır. Eşitlik 22 'in zamana göre türevi ise Eşitlik 23 'teki gibi elde edilir.

$$
\begin{aligned}
& \Rightarrow \dot{V}=r^{T} M \dot{r}+\tilde{z} \dot{\tilde{z}}+\tilde{\phi}^{T} \Gamma_{\phi}^{-1} \dot{\tilde{\phi}}+\tilde{\theta}^{T} \Gamma^{-1} \dot{\tilde{\theta}} \\
& +\frac{1}{\gamma_{1}} \tilde{\sigma}_{a} \dot{\tilde{\sigma}}_{a}+\frac{1}{\gamma_{2}} \tilde{\sigma}_{0} \dot{\tilde{\sigma}}_{0}+\frac{1}{\gamma_{3}} a_{0}^{2} \tilde{\sigma}_{1} \dot{\tilde{\sigma}}_{1}+P
\end{aligned}
$$

$$
\begin{aligned}
& P=\sigma_{a}\left(\tilde{z}-\xi_{1}\right)\left(\dot{\bar{z}}-\dot{\xi}_{1}\right) \\
& +\sigma_{0}\left(\tilde{z}-\xi_{2}\right)\left(\dot{\tilde{z}}-\dot{\xi}_{2}\right)+\sigma_{1} a_{0}\left(\tilde{z}-\xi_{3}\right)\left(\dot{\tilde{z}}-\dot{\xi}_{3}\right)
\end{aligned}
$$

Adaptasyon kuralları ve yardımcı filtreler aşağıdaki gibi seçilirse ve Eşitlik 23 ve Eşitlik 24'te yerlerine konulursa, Eşitlik 22 'nin zamana göre türevi Eşitlik 26'daki formu alacaktır.

$$
\begin{gathered}
\dot{\tilde{\phi}}=-\dot{\hat{\phi}}=-\Gamma_{\phi} Y^{T} r \\
\dot{\tilde{\theta}}=-\dot{\hat{\theta}}=\Gamma \rho^{T} H^{T} r \\
\dot{\tilde{\sigma}}_{a}=-\dot{\hat{\sigma}}_{a}=\gamma_{1}\left(\hat{z}+\xi_{1}\right) H^{T} r \\
\dot{\tilde{\sigma}}_{0}=-\dot{\hat{\sigma}}_{0}=\gamma_{2} v\left(\hat{z}+\xi_{2}\right) H^{T} r \\
\dot{\tilde{\sigma}}_{1}=-\dot{\hat{\sigma}}_{1}=-\frac{\gamma_{3}}{a_{0}}\left|\dot{x}_{r}\right|\left(\hat{z}+\xi_{3}\right) H^{T} r \\
\dot{\xi}_{1}=-a_{0}\left|\dot{x}_{r}\right| \xi_{1}-H^{T} r \\
\dot{\xi}_{2}=-a_{0}\left|\dot{x}_{r}\right| \xi_{2}-v H^{T} r \\
\dot{\xi}_{3}=-a_{0}\left|\dot{x}_{r}\right| \xi_{3}+\left|\dot{x}_{r}\right| H^{T} r \\
\dot{V}=-r^{T} K r-a_{0}\left|\dot{x}_{r}\right| \tilde{z}^{2}-\sigma_{a} a_{0}\left|\dot{x}_{r}\right|\left(\tilde{z}-\xi_{1}\right)^{2} \\
-\sigma_{0} a_{0}\left|\dot{x}_{r}\right|\left(\tilde{z}-\xi_{2}\right)^{2}-\sigma_{1} a_{0}^{2}\left|\dot{x}_{r}\right|\left(z-\xi_{3}\right)^{2}
\end{gathered}
$$

Eşitlik 26 incelendiğinde görülebilir ki eğer $\mathrm{K}$ matrisi pozitif tanımlı olarak seçilirse Eşitlik 26 her zaman negatif olacaktır. Sonuç olarak Barbalat'ın yardımcı önermesini kullanarak [23], zamanın sonsuza giderken hata dinamiğinin sıfira gideceğini söyleyebiliriz.

\section{ARAŞTIRMA VE BULGULAR}

Bu çalışmada, modellemeler ile YAKS'ye uygulanan uyarlamalı kontrol algoritmas1 Matlab-Simulink ortamında gerçekleştirilmiştir. Lugre MR sönümleyici modeline ait parametre değerleri için referans [18] kullanılmıştır. Yapısal sistemin doğal frekanslarının toplamından elde edilen tahrik girişi performans analizinde kullanılmış ve yapısal sisteme ait yer değiştirme ve ivme cevapları incelenmiştir. 1 . kat ve 10 . katların yer değiştirme cevapları Şekil 4'te, tüm katlara ait maksimum yer değiştirmeler ve yer değiştirme RMS değerleri Şekil 5 'te görülmektedir.
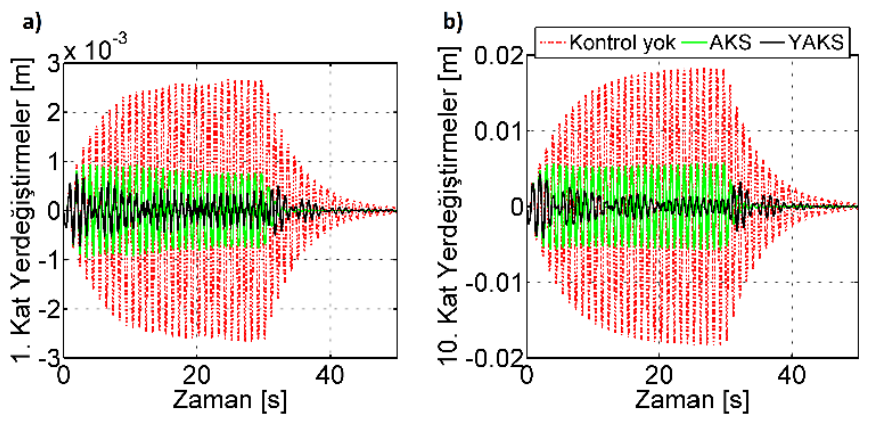

Şekil 4 Yer değiştirmeler a) 1. kat b) 10. kat

Şekil 4 Displacements a) 1. floor b) 10. floor

Eğriler incelendiğinde pasif veya yarı aktif kontrolün olmadığ duruma göre AKS'nin ve YAKS'nin ikisinin de başarılı olduğu açıkça görülmektedir. Fakat en iyi performansı YAKS'nin sergilediği görülmektedir. Şekil 4'te ise, beklenildiği gibi kütle binada sönümleyicinin bulunmadığı duruma göre AKS 
ve YAKS'nin katlara ait yer değiştirme cevaplarının performansı, üst katlara çıkıldıkça artmıştır.
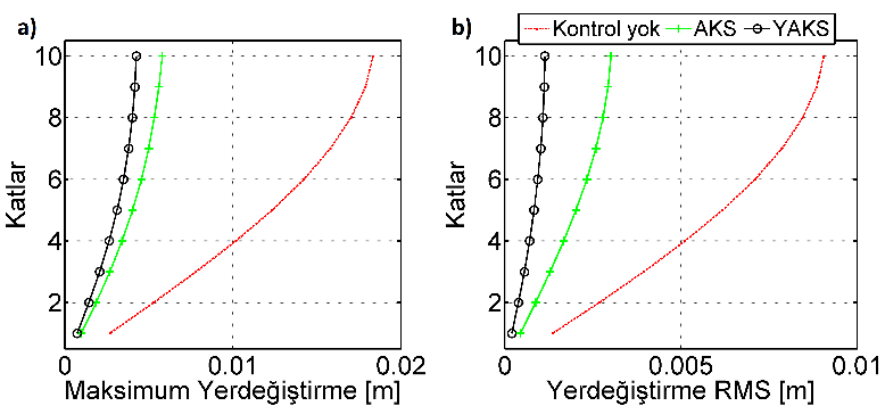

Şekil 5 Tüm katların maksimum cevapları a) yer değiştirmeler b) yer değiştirme RMS'ler

Figure 6 Maximum responses of all floors a) dispalcements b) RMS of displacements

Şekil 6'da sistemin 1. kat ve 10. katlarının ivme cevapları, Şekil 7'de tüm katların maksimum ivmeleri ve ivme RMS değerleri, Şekil 8'de ise her iki kontrol durumunun, kontrol uygulamasının olmadığı duruma göre maksimum yer değiştirmelerine ve maksimum ivmelerine ait yüzde iyileşme performans değerleri gösterilmektedir. Eğriler incelendiğinde, her iki kontrol uygulamasının da yer değiştirmenin yanında sistemin ivme cevaplarını etkili bir şekilde bastırdığ görülmektedir. Yer değiştirme cevaplarında olduğu gibi ivme cevaplarında da uyarlamalı kontrol etkisindeki YAKS'nin performansının AKS'den daha yüksek olduğu görülmektedir.
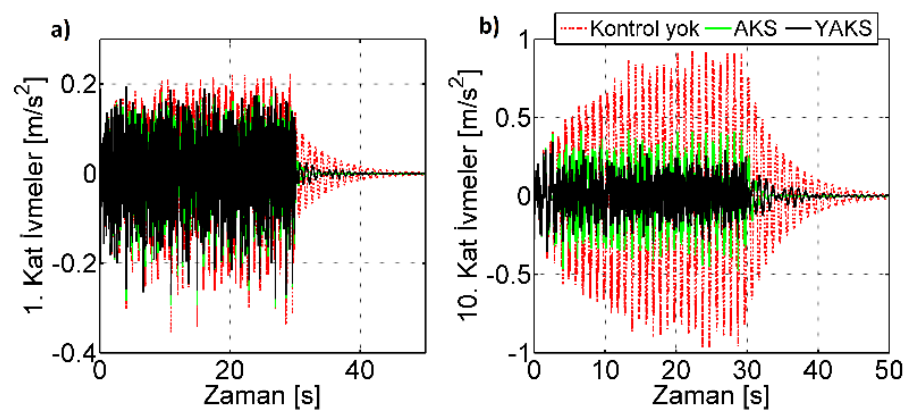

Şekil 7 İvmeler a) 1. kat b) 10. kat

Figure 8 Accelerations a) 1. floor b) 10. floor
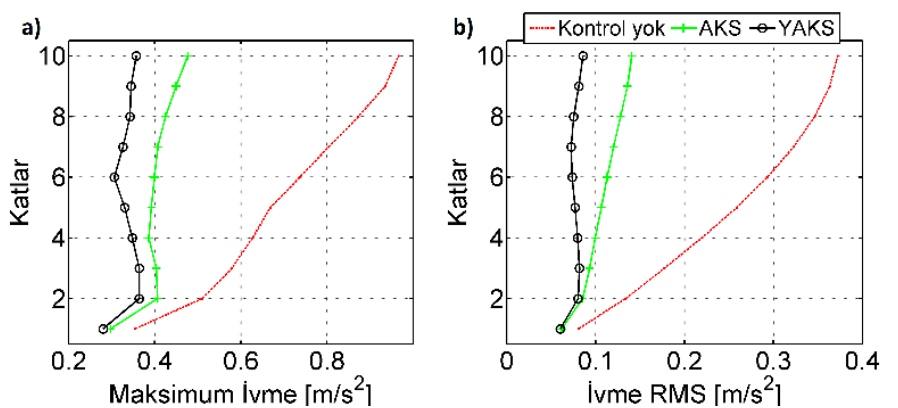

Şekil 9 Tüm katların maksimum cevapları a) ivmeler b) ivme RMS'ler

Figure 7 Maximum responses of all floors a) accelerations b) RMS of accelerations

Şekil 6 (a)'da ve Şekil 7'de, 1. kata ait ivme cevaplarında her iki kontrol durumunun da performanslarının birbirlerine çok yakın olduğu görülmektedir. MR sıvısının mili saniye gibi çok küçük zaman aralıklarında akışkanlığının değişmesi, anlık olarak MR damperde kilitlenmelere neden olabilir. Bu da YAKS'deki ivme cevaplarında performans düşüklüğü olarak karşımıza çıkar. Asıl odaklanılan yer değiştirmelerdeki yüksek performanslar dikkate alındığında, ivme cevaplarındaki bu davranış kabul edilebilir bir durumdur.
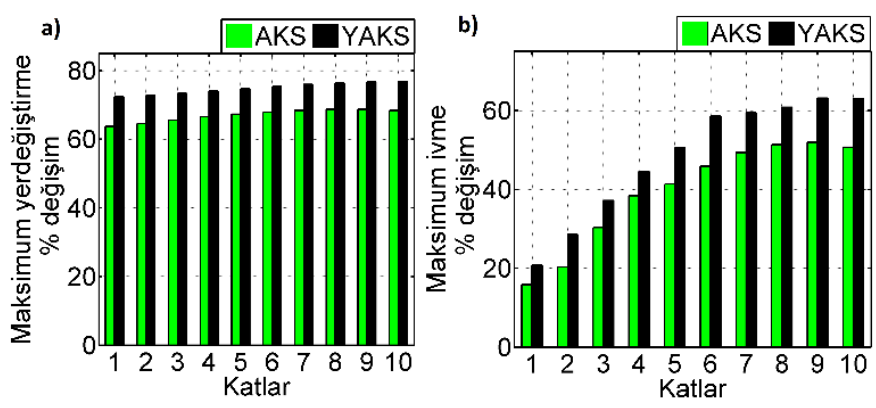

Şekil 10 Tüm katların maksimum yüzde değişim miktarları a) yer değiştirmeler b) ivmeler

Figure 11 Maximum percentage changes of all floors a) dispalcements b) accelerations

\section{Sonuçlar}

Bu çalışmada, YAKS'lerin bir bina modeli üzerindeki performansı sayısal simülasyonlar ile incelenmiştir. YAKS'nin yarı aktifliğini sağlayan akıllı kontrol elemanı olarak MR sönümleyici kullanılmıştır. MR sönümleyici nonlineer histerezis karaktere sahiptir. Ayrıca sistem parametrelerinde çalışma sırasında bazı değişiklikler olabileceği bilinmektedir. Bu yüzden sistemin nonlineer yapısı ve parametrik belirsizlerin üstesinden gelebilmek amacıyla MR sönümleyiciye gerekli olan gerilimi belirlemesi için nonlineer uyarlamalı kontrolör tasarımı yapılmıştır. Sonuçlar, zamana göre katların yer değiştirmeleri, ivmeleri ve RMS değerleri kullanılarak incelenmiştir. Sonuçlar genel olarak, sistemde AKS ve YAKS bulunmasının, sistemde herhangi bir kütle sönümleyicisi bulunmayan duruma göre, bina yer değiştirmelerini ve ivmelerini önemli ölçüde azalttığını göstermiştir. Ayrıca aktif kütle sönümleyicilerine göre daha az maliyetli, daha güvenli ve bir akü ile çalışabilecek kadar düşük enerji tüketimine sahip MR sönümleyicinin uygun kontrol yöntemi kullanılmasıyla AKS'den daha iyi performans gösterdiği görülmüştür.

\section{Kaynakça}

[1] Guclu, R., \& Sertbas, A. (2005). Evaluation of sliding mode and proportional-integral-derivative controlled structures with an active mass damper. Journal of Vibration and Control, 11(3), 397-406.

[2] Guclu, R., \& Yazici, H. (2008). Vibration control of a structure with ATMD against earthquake using fuzzy logic controllers. Journal of Sound and Vibration, 318(1-2), 3649.

[3] Guclu, R., \& Yazici, H. (2009). Self-tuning fuzzy logic control of a non-linear structural system with ATMD against earthquake. Nonlinear Dynamics, 56(3), 199-211.

[4] Guclu, R., \& Yazici, H. (2009). Seismic-vibration mitigation of a nonlinear structural system with an ATMD through a fuzzy PID controller. Nonlinear Dynamics, 58(3), 553-564.

[5] Aggümüş, H. (2020) "Simülasyon çevriminde donanım yöntemiyle yarı aktif ayarlı kütle sönümleyicilerinin performans analizi," Doktora tezi, YTÜ Fen Bilimleri Enstitüsü,2020. 
[6] Aldemir, U. (2003). Optimal control of structures with semiactive-tuned mass dampers. Journal of sound and vibration, 266(4), 847-874.

[7] Lin, P. Y., Chung, L. L., \& Loh, C. H. (2005). Semiactive control of building structures with semiactive tuned mass damper. Computer-Aided Civil and Infrastructure Engineering, 20(1), 35-51.

[8] Tse, K. T., Kwok, K. C. S., Hitchcock, P. A., Samali, B., \& Huang, M. F. (2007). Vibration control of a wind-excited benchmark tall building with complex lateral-torsional modes of vibration. Advances in Structural Engineering, 10(3), 283-304.

[9] Bathaei, A., Zahrai, S. M., \& Ramezani, M. (2018). Semiactive seismic control of an 11-DOF building model with TMD+ MR damper using type-1 and-2 fuzzy algorithms. Journal of Vibration and Control, 24(13), 2938-2953.

[10] Aly, A. M. (2015). Control of wind-induced motion in highrise buildings with hybrid TM/MR dampers. Wind \& structures, 21(5), 565-595.

[11] Setareh, M., Ritchey, J. K., Murray, T. M., Koo, J. H., \& Ahmadian, M. (2007). Semiactive tuned mass damper for floor vibration control. Journal of structural engineering, 133(2), 242-250.

[12]Lin, P. Y., Lin, T. K., \& Hwang, J. S. (2013). A semi-active mass damping system for low-and mid-rise buildings. Earthquakes and Structures, 4(1), 63-84.

[13]Weber, F., Distl, H., Fischer, S., \& Braun, C. (2016, December). MR damper controlled vibration absorber for enhanced mitigation of harmonic vibrations. In Actuators (Vol. 5, No. 4, p. 27). Multidisciplinary Digital Publishing Institute.

[14]Aggumus, H., \& Cetin, S. (2018). Experimental investigation of semiactive robust control for structures with magnetorheological dampers. Journal of Low Frequency Noise, Vibration and Active Control, 37(2), 216-234.

[15]Aggumus, H., \& Guclu, R. (2020, September). Robust Hळ control of STMDs used in structural systems by hardware in the loop simulation method. In Actuators (Vol. 9, No. 3, p. 55). Multidisciplinary Digital Publishing Institute.

[16]Aggümüş, H. Güçlü R. (2020) "Yar1-aktif kütle sönümleyici kullanarak bina titreşimlerinin hibrit simülasyon yöntemiyle azaltılması," 3. Uluslararası Bilim, Teknoloji ve Sosyal Bilimlerde Güncel Gelişmeler Sempozyumu, Adana, Türkiye, 19-20 Haziran 2020, cilt.2, no.34, ss.391-397.

[17]Paksoy, M., \& Metin, M. (2019). Nonlinear semi-active adaptive vibration control of a half vehicle model under unmeasured road input. Journal of Vibration and Control, 25(18), 2453-2472.

[18]Paksoy, M., \& Metin, M. (2020). Nonlinear adaptive semiactive control of a half-vehicle model via hardware in the loop simulation. Turkish Journal of Electrical Engineering \& Computer Sciences, 28(3), 1612-1630.

[19]Nassani, D. E. \& Ali, K. (2020). Lateral Load Resisting Systems in High-Rise Reinforced Concrete Buildings. Avrupa Bilim ve Teknoloji Dergisi, (20), 397-403 .

[20]Sadek, F., Mohraz, B., Taylor, A. W., \& Chung, R. M. (1997). A method of estimating the parameters of tuned mass dampers for seismic applications. Earthquake Engineering \& Structural Dynamics, 26(6), 617-635.

[21]Spencer Jr, B., Dyke, S. J., Sain, M. K., \& Carlson, J. (1997). Phenomenological model for magnetorheological dampers. Journal of engineering mechanics, 123(3), 230238.
[22]Sakai, C., Ohmori, H., \& Sano, A. (2003). Modeling of MR damper with hysteresis for adaptive vibration control. In 42nd IEEE International Conference on Decision and Control (IEEE Cat. No. 03CH37475) (Vol. 4, pp. 38403845). IEEE.

[23]Krstic, M., Kokotovic, P. V., \& Kanellakopoulos, I. (1995). Nonlinear and adaptive control design. John Wiley \& Sons, Inc.

[24] A. Turan, C. Onat, M. Sahin, (2019). 'Active Vibration Suppression of A Smart Beam Via PID Controller Designed Through Weighted Geometric Center Method' 10th Ankara International Aerospace Conference, 18-20 September, METU, Ankara TURKEY. 